\title{
Development of Underwater Motion Capture System for Space Suit Mobility Assessment
}

Human Factors and Ergonomics Society 2017

Yaritza Bernal

K. Han Kim, PhD

Elizabeth Benson

Sarah Jarvis

Lauren Harvill

lan Meginnis

Sudhakar Rajulu, PhD
Geologics Corp.

Leidos

MEI Technologies, Inc.

MEI Technologies, Inc.

Leidos

NASA Johnson Space Center

NASA Johnson Space Center 


\section{Background}

- Space suits are an essential component for extravehicular activity (EVA) operations.

- Pressurized suits can significantly affect the motions and performance of the crewmember.

- Understanding kinematic patterns and variations is essential for hardware design improvements and for EVA planning.

- An underwater setting has been used by NASA for the simulation of microgravity environment for suited testing and training.

- Motion capture underwater has been a challenger so far.

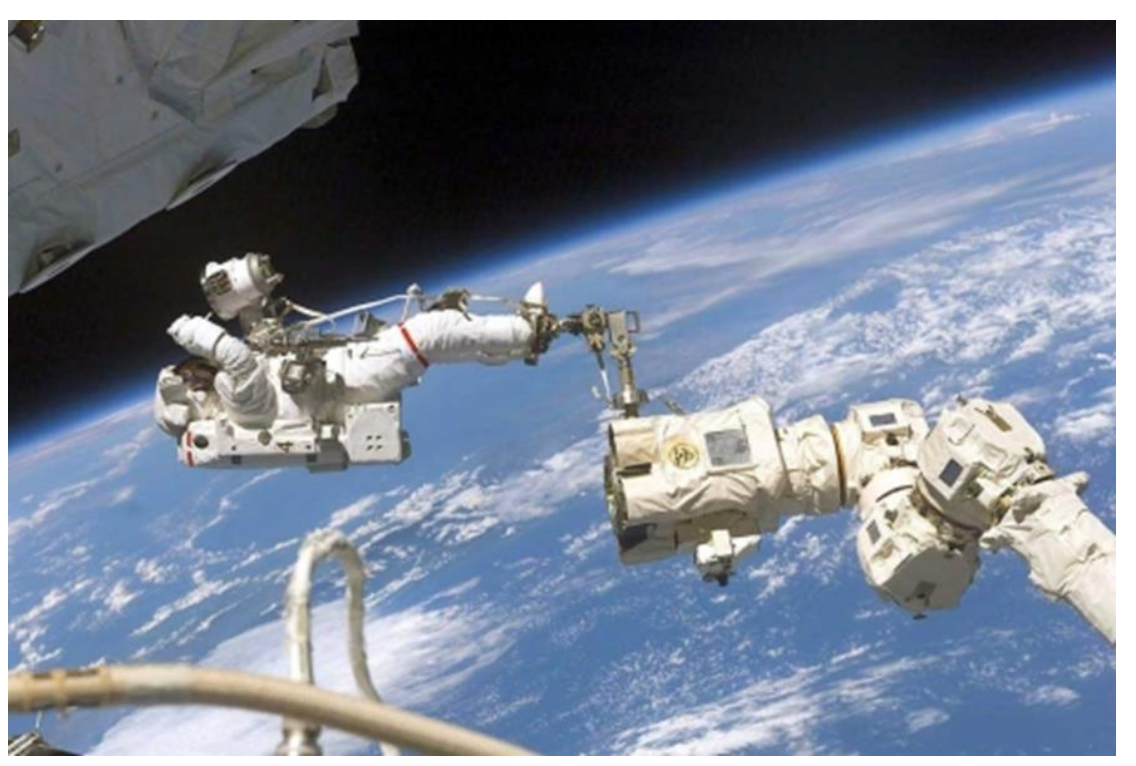




\section{Suited Reach Envelope: Previous Work}

- Data generated using computer-based anthropometric model.

- Planar tracking of hand positions in a dive tank.
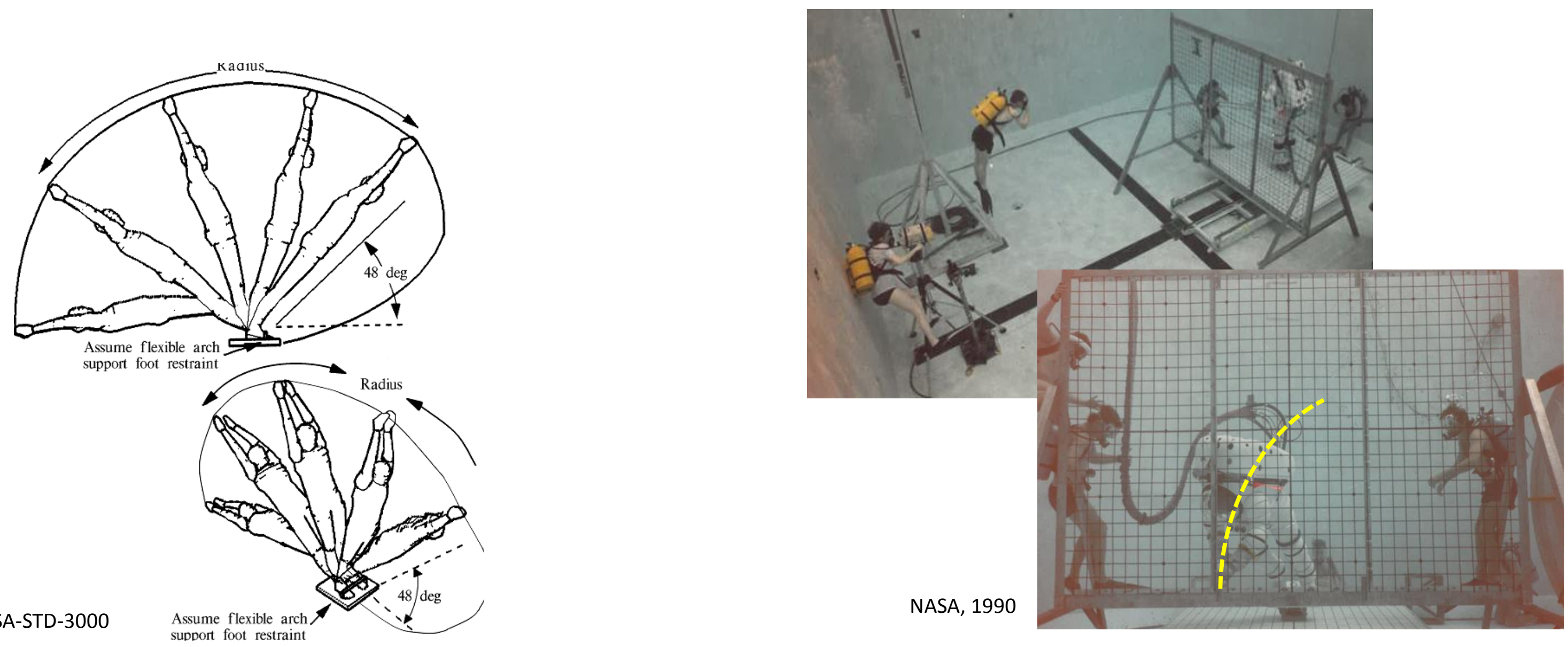


\section{Suited Mobility Measurements in Simulated Microgravity}

- The aim of the study was a) to deploy a relatively inexpensive and effective methodology to study motion analysis underwater and $b$ ) to use this methodology to evaluate the performance of current and new prototype suits.

- Testing was performed at the Neutral Buoyancy Laboratory (NBL) at NASA Johnson Space Center (62 m x 31m x 12.3m diving tank).

- This study compared the 3-D hand traces between the current space suit and a new prototype space suit.

- Rather than using traditional motion capture techniques, this method allowed us to capture the volume, area, and shape of reach traces that would serve as metrics of the mobility performance of the suits.
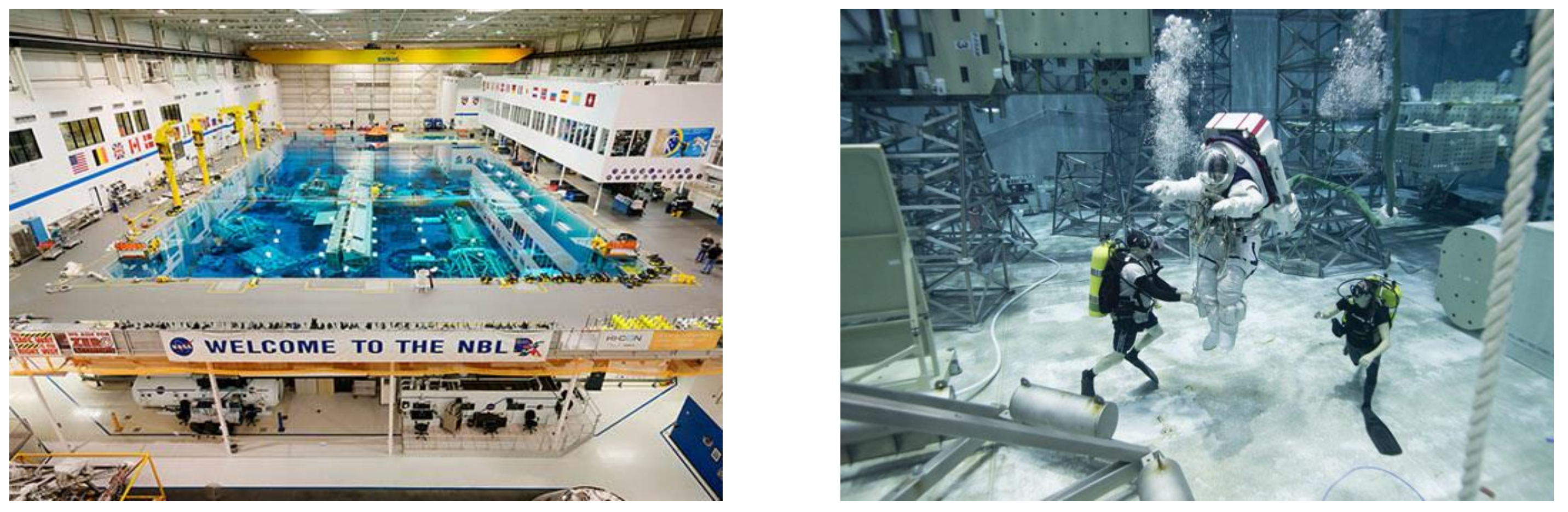


\section{Underwater Motion Capture System Development}

- A new system was developed using optical triangulation of markers using multiple camera images.

- A proof-of-concept system was first developed and tested on dry land before underwater deployment.

- Four GoPro Hero 4 cameras were used (1920 x 1080 resolution at 30 frames/s).

- Each camera and housing combination was calibrated using a checkerboard to correct the optical distortions.
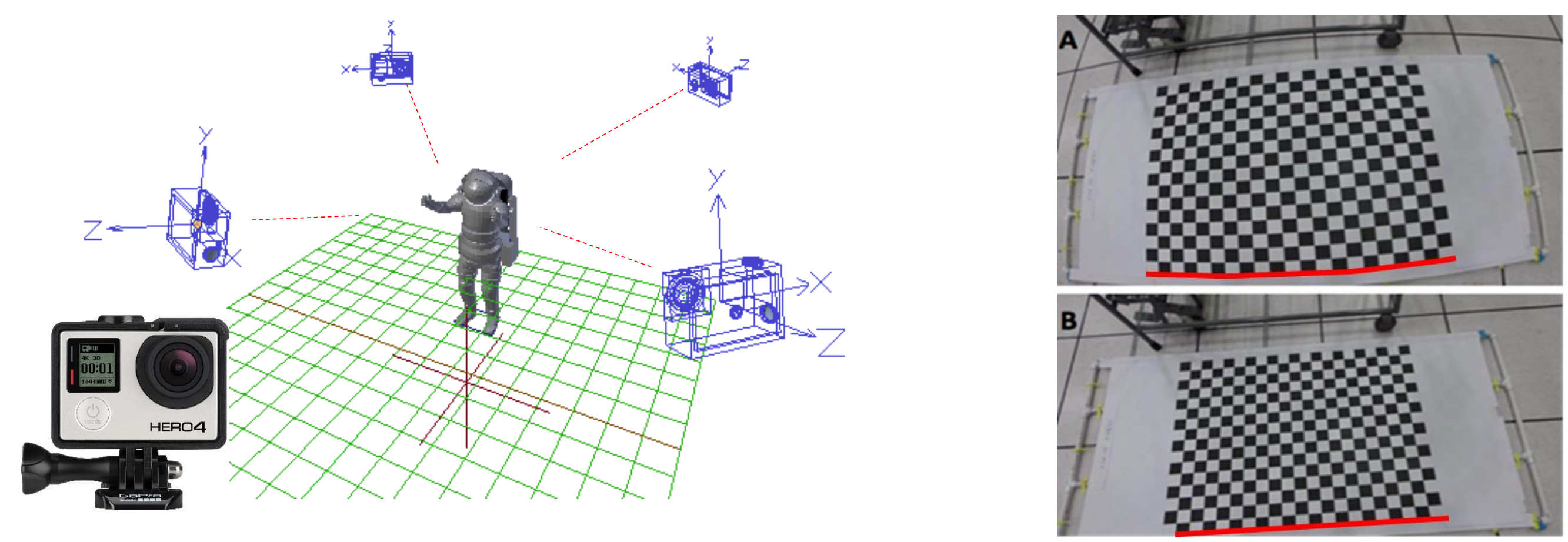


\section{System Development and "Dry-Land" Validation}

- Camera positions were determined using a global coordinate reference frame.

- A hand-held wand with reflective markers made sweeping motions.

- GoPro and Vicon systems were synchronized and simultaneously recorded the marker motions.

- Markers on the images were tracked using a pattern-matching algorithm in 3-D modeling software Blender.

- 2-D image coordinates were taken to triangulate the 3-D coordinates using a Direct Linear Transform algorithm in OpenCV.

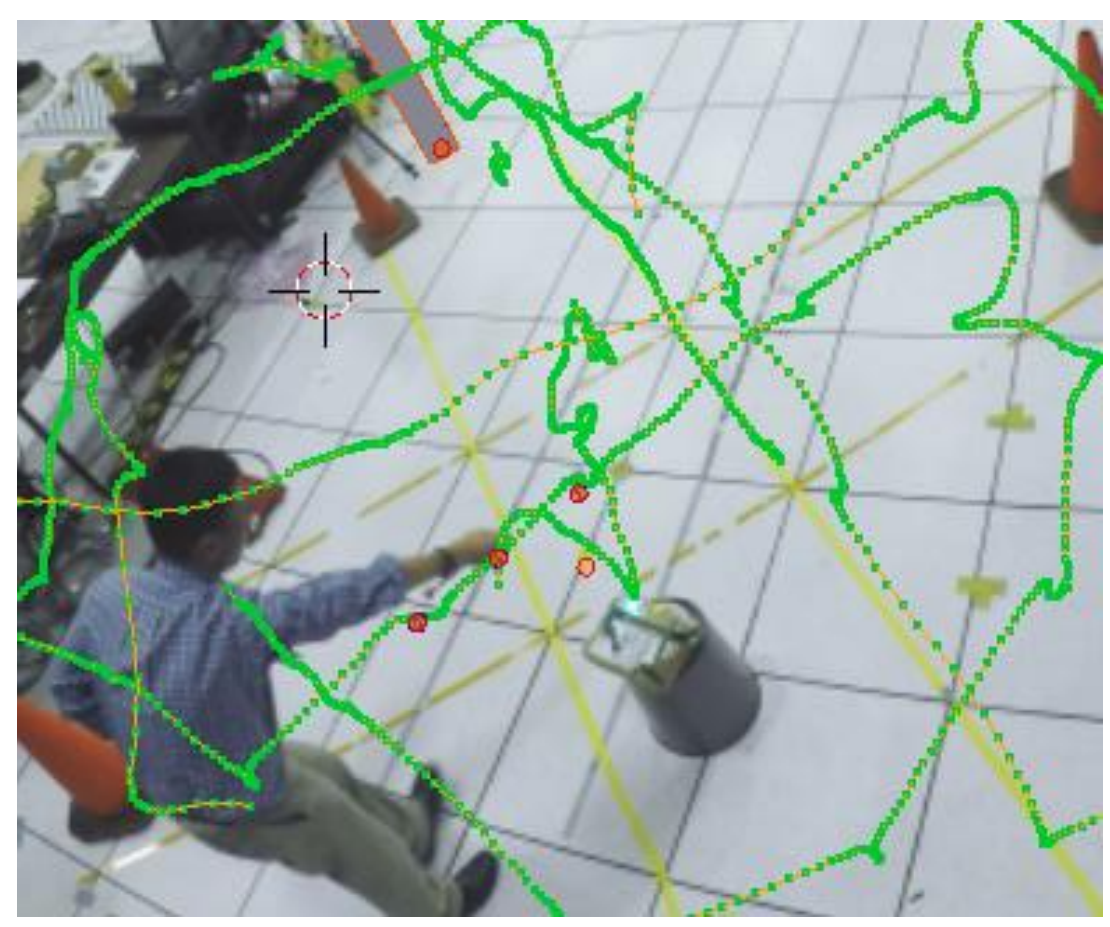




\section{System Validation Results}

- Overall, 3-D marker traces were in good agreement between the GoPro and Vicon system (Gold Standard).

- Linear distance error between GoPro and Vicon-estimated marker positions was $1.90 \mathrm{~cm}$ RMSE (SD $1.41 \mathrm{~cm}$ ).

- Measurement errors were uniformly distributed within the calibration volume, but increased for points beyond the calibration

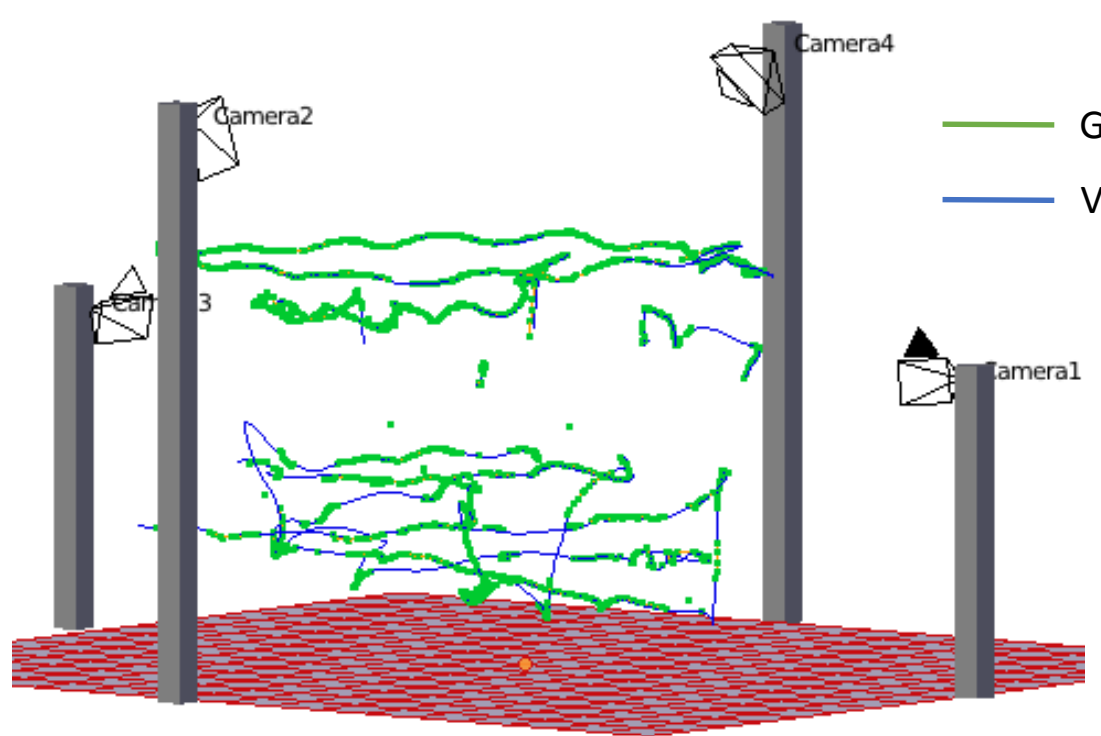
volume.
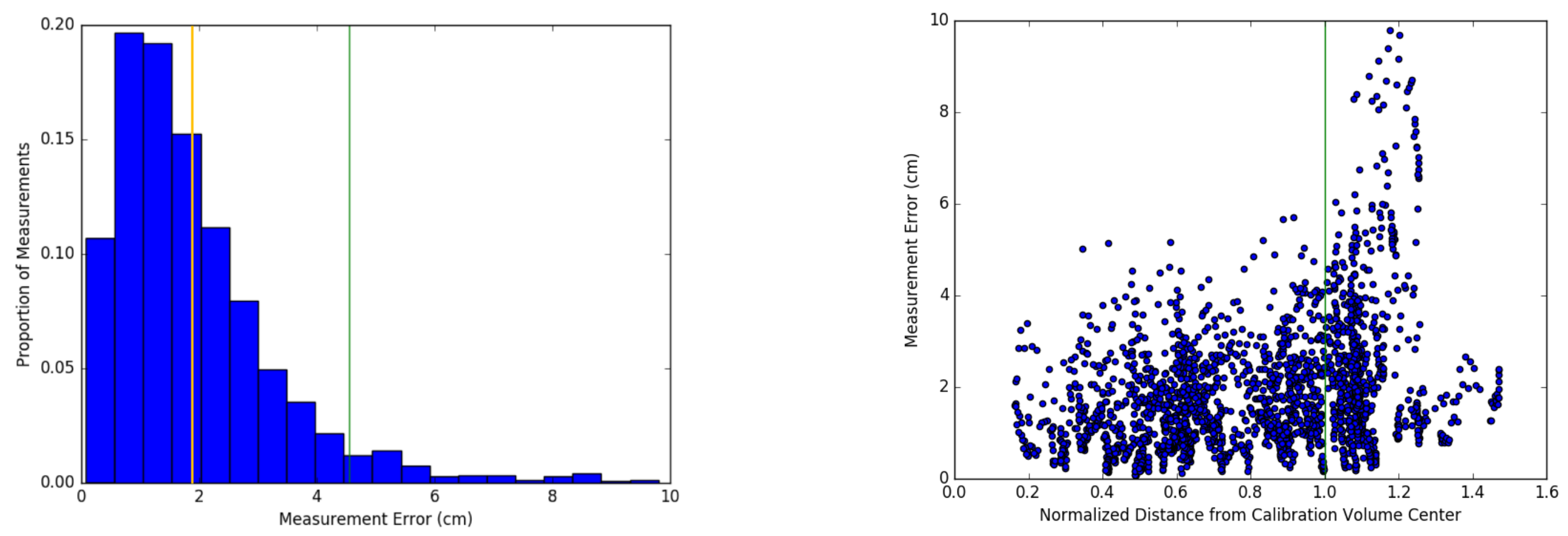


\section{Underwater System Deployment}

- Each GoPro camera and housing combination was optically calibrated again underwater with divers.

- Capture volume was set up on a $5.5 \mathrm{~m} \times 4.8 \mathrm{~m}$ surface area.

- Camera positions were determined in a global coordinate reference frame with 32 spherical markers.

- A dive emergency strobe light was used to synchronize all 4 cameras.
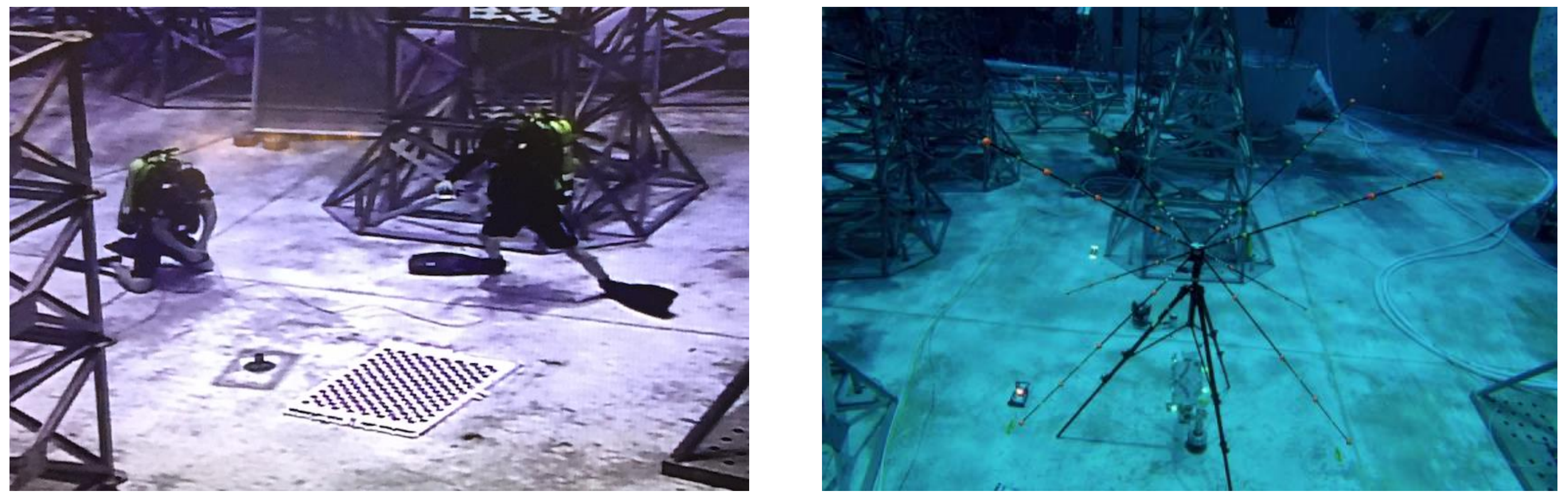


\section{Suited Reach Motion Measurements}

- 5 crewmembers and 2 suit engineers participated.

- Current space suit Extravehicular Mobility Unit (EMU) and new prototype space suit (Z-2) were tested.

- The suited subject was placed on an articulating portable foot restraint (APFR).

- The subject performed prescribed reach motions:

- Isolated Arm Motions

- Right arm

- Left arm

- Whole-Body Reach Motions

- Vertical sweeps at $0^{\circ}, 45^{\circ}, 90^{\circ}$ left and right

- Horizontal sweeps at overhead, shoulder, waist, and below waist level

- Free motions

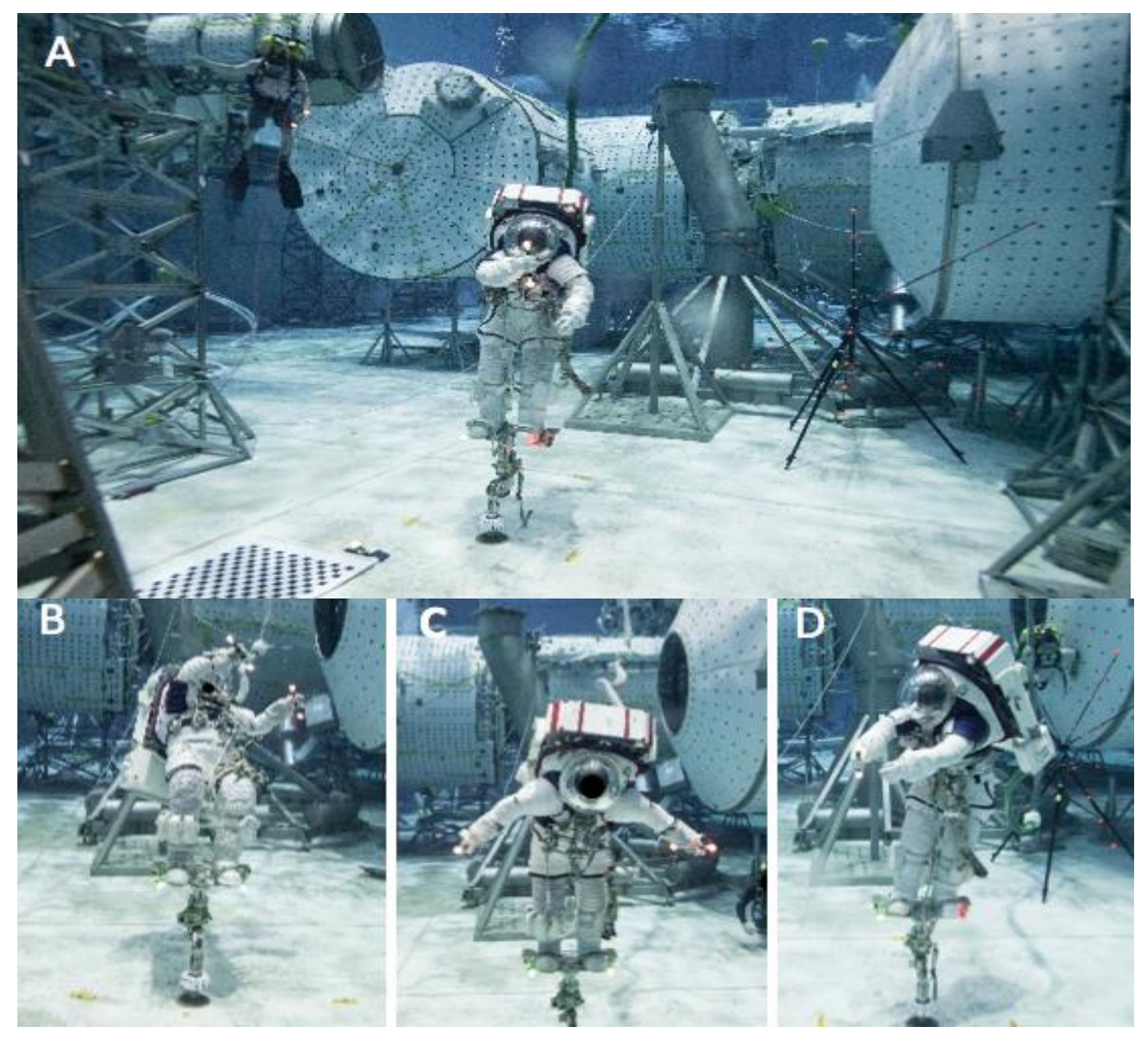




\section{Hand Tracking using Active Markers}

- Subject held marker wands in the hands.

- A dive LED light was attached inside a ping-pong ball diffuser at each end of the wand.

- Markers were tracked and triangulated in the capture volume without an identifiable "missing" zone.

- $100 \%$ of frames were visible in the isolated arm motions, $96.2 \%$ of frames were visible in the whole-body motions.
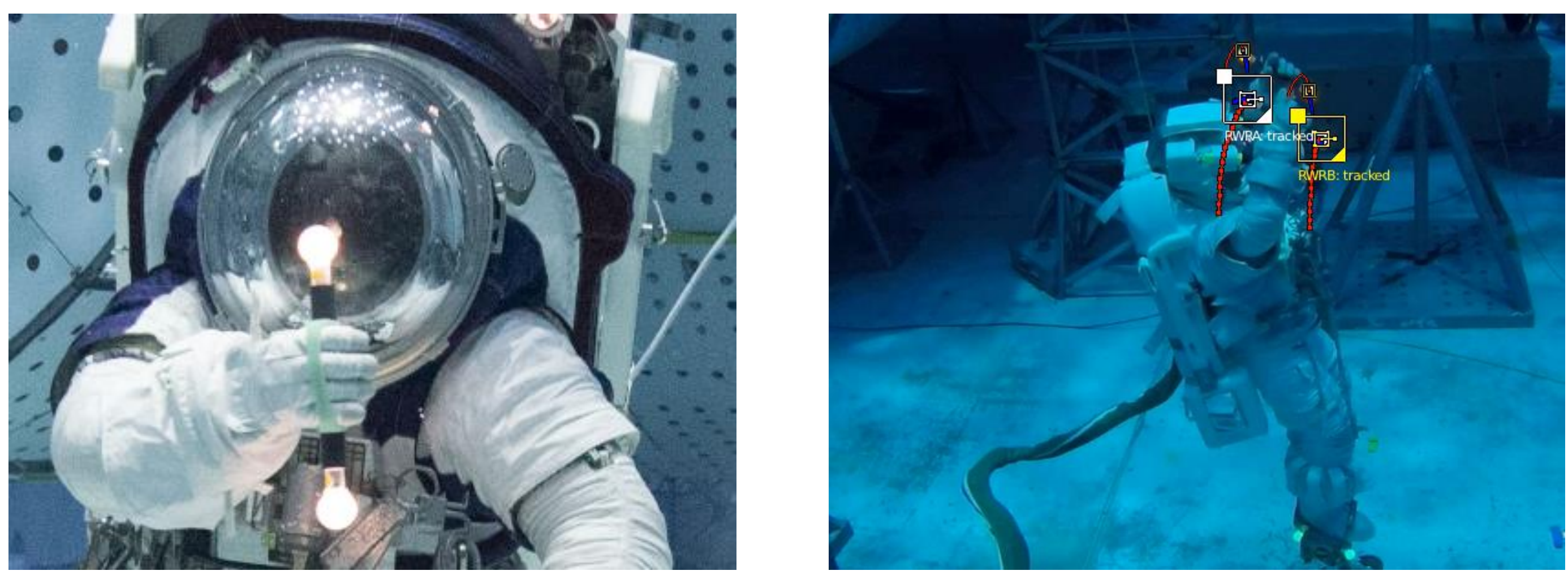


\section{3-D Hand Traces for Suit Mobility Metrics: Isolated Arm-Hand Reach Tasks}

- 3-D hand traces were acquired from isolated arm-hand reach tasks.

- Shape and area of the reach envelope provided metrics for upper extremity mobility.
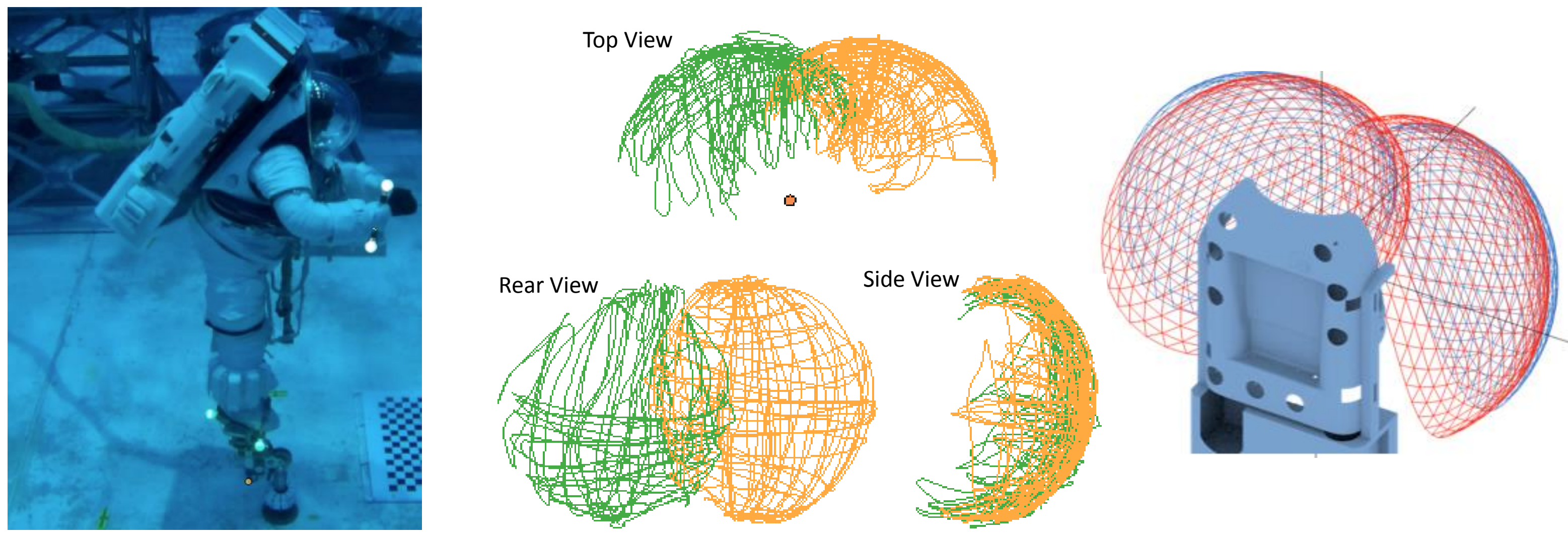


\section{3-D Hand Traces for Suit Mobility Metrics (cont.): Whole-Body Reach Tasks}

- Whole-body reach span measurements were estimated.

- Reach envelopes were also estimated and compared for different suit types.
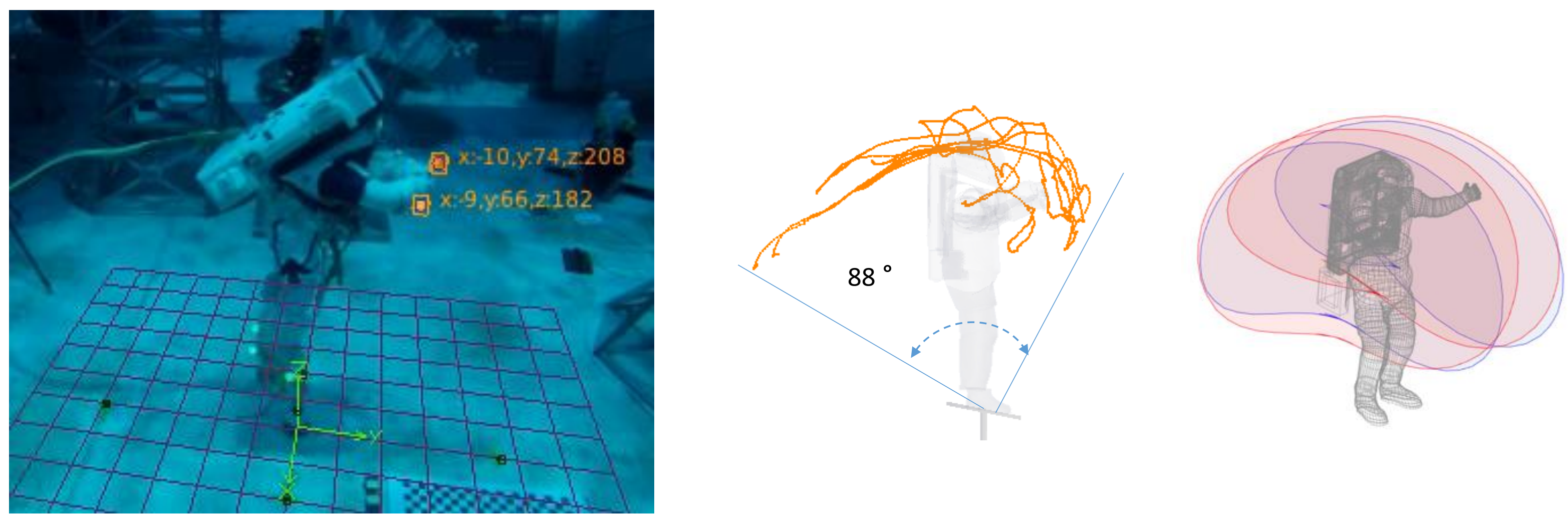


\section{Discussion \& Conclusion}

\section{Advantage of New System}

- Utilizes off-the-shelf equipment, thus inexpensive and as reliable as a standard robust system.

- Motion tracking and analysis procedures were efficient with open source software tools.

- Accuracy level adequate for the context of suit performance testing of "gross" motion trajectories.

- Active LED markers enhanced detectability and tracking accuracy in the underwater environment.

\section{Limitation}

- Difficult to install and secure the camera positions underwater.

- Marker mislabeling in automatic tracking remains as an unresolved issue. Light is absorbed by water therefore after a depth of $20 \mathrm{ft}$. colors are hard to discriminate.

\section{Conclusion}

The new system provided a useful and substantially less expensive method of 3-D motion capture. The new method can be used in extreme ergonomic environments, unlike traditional motion capture. 


\title{
Contact Information
}

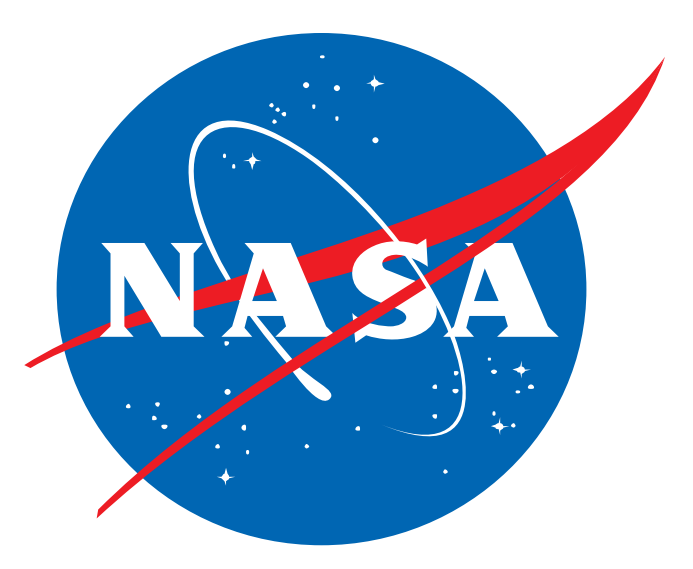

\author{
Yaritza Bernal \\ Geologics Corp. \\ Anthropometry and Biomechanics Facility \\ NASA Johnson Space Center \\ Email: Yaritza.Bernal@nasa.gov \\ Sudhakar Rajulu, PhD \\ Anthropometry and Biomechanics Facility \\ NASA Johnson Space Center \\ Email: Sudhakar.Rajulu-1@nasa.gov
}

